\title{
Data dependence results of new multi-step and S-iterative schemes for contractive-like operators
}

\author{
Faik Gürsoy' ${ }^{1}$ Vatan Karakaya ${ }^{2 *}$ and Billy E Rhoades ${ }^{3}$
}

\section{"Correspondence:}

vkkaya@yildiz.edu.tr;

vkkaya@yahoo.com

${ }^{2}$ Department of Mathematical

Engineering, Faculty of Chemical and Metallurgical Engineering, Yildiz

Technical University, Davutpasa Campus, Esenler, Istanbul, 34210,

Turkey

Full list of author information is available at the end of the article

\begin{abstract}
In this paper, we prove that the convergence of a new iteration and S-iteration can be used to approximate the fixed points of contractive-like operators. We also prove some data dependence results for these new iteration and S-iteration schemes for contractive-like operators. Our results extend and improve some known results in the literature.

MSC: Primary $47 \mathrm{H} 10$

Keywords: new multi-step iteration; S-iteration; data dependence; contractive-like operator
\end{abstract}

\section{Introduction}

Contractive mappings and iteration procedures are some of the main tools in the study of fixed point theory. There are many contractive mappings and iteration schemes that have been introduced and developed by several authors to serve various purposes in the literature of this highly active research area, viz., [1-12] among others.

Whether an iteration method used in any investigation converges to a fixed point of a contractive type mapping corresponding to a particular iteration process is of utmost importance. Therefore it is natural to see many works related to the convergence of iteration methods such as [13-22].

Fixed point theory is concerned with investigating a wide variety of issues such as the existence (and uniqueness) of fixed points, the construction of fixed points, etc. One of these themes is data dependency of fixed points. Data dependency of fixed points has been the subject of research in fixed point theory for some time now, and data dependence research is an important theme in its own right.

Several authors who have made contributions to the study of data dependence of fixed points are Rus and Muresan [23], Rus et al. [24, 25], Berinde [26], Espínola and Petruşel [27], Markin [28], Chifu and Petruşel [29], Olantiwo [30, 31], Şoltuz [32, 33], Şoltuz and Grosan [34], Chugh and Kumar [35] and the references therein.

This paper is organized as follows. In Section 1 we present a brief survey of some known contractive mappings and iterative schemes and collect some preliminaries that will be used in the proofs of our main results. In Section 2 we show that the convergence of a new multi-step iteration, which is a special case of the Jungck multistep-SP iterative process defined in [36], and S-iteration (due to Agarwal et al.) can be used to approximate the fixed

\section{Springer}

(c) 2013 Gürsoy et al.; licensee Springer. This is an Open Access article distributed under the terms of the Creative Commons Attribution License (http://creativecommons.org/licenses/by/2.0), which permits unrestricted use, distribution, and reproduction in any medium, provided the original work is properly cited. 
points of contractive-like operators. Motivated by the works of Şoltuz [32, 33], Şoltuz and Grosan [34], and Chugh and Kumar [35], we prove two data dependence results for the new multi-step iteration and S-iteration schemes by employing contractive-like operators.

As a background of our exposition, we now mention some contractive mappings and iteration schemes.

In [37] Zamfirescu established an important generalization of the Banach fixed point theorem using the following contractive condition. For a mapping $T: E \rightarrow E$, there exist real numbers $a, b, c$ satisfying $0<a<1,0<b, c<1 / 2$ such that, for each pair $x, y \in X$, at least one of the following is true:

$$
\begin{cases}\left(\mathrm{z}_{1}\right) & \|T x-T y\| \leq a\|x-y\|, \\ \left(\mathrm{z}_{2}\right) & \|T x-T y\| \leq b(\|x-T x\|+\|y-T y\|), \\ \left(\mathrm{z}_{3}\right) & \|T x-T y\| \leq c(\|x-T y\|+\|y-T x\|) .\end{cases}
$$

A mapping $T$ satisfying the contractive conditions $\left(\mathrm{z}_{1}\right),\left(\mathrm{z}_{2}\right)$ and $\left(\mathrm{z}_{3}\right)$ in (1.1) is called a Zamfirescu operator. An operator satisfying condition $\left(\mathrm{z}_{2}\right)$ is called a Kannan operator, while the mapping satisfying condition $\left(\mathrm{z}_{3}\right)$ is called a Chatterjea operator. As shown in [13], the contractive condition (1.1) leads to

$$
\left\{\begin{array}{l}
\left(\mathrm{b}_{1}\right) \quad\|T x-T y\| \leq \delta\|x-y\|+2 \delta\|x-T x\| \text { if one uses }\left(\mathrm{z}_{2}\right), \text { and } \\
\left(\mathrm{b}_{2}\right) \quad\|T x-T y\| \leq \delta\|x-y\|+2 \delta\|x-T y\| \text { if one uses }\left(\mathrm{z}_{3}\right),
\end{array}\right.
$$

for all $x, y \in E$, where $\delta:=\max \left\{a, \frac{b}{1-b}, \frac{c}{1-c}\right\}, \delta \in[0,1)$, and it was shown that this class of operators is wider than the class of Zamfirescu operators. Any mapping satisfying condition $\left(b_{1}\right)$ or $\left(b_{2}\right)$ is called a quasi-contractive operator.

Extending the above definition, Osilike and Udomene [20] considered operators $T$ for which there exist real numbers $L \geq 0$ and $\delta \in[0,1)$ such that for all $x, y \in E$,

$$
\|T x-T y\| \leq \delta\|x-y\|+L\|x-T x\| .
$$

Imoru and Olantiwo [38] gave a more general definition: An operator $T$ is called a contractive-like operator if there exists a constant $\delta \in[0,1)$ and a strictly increasing and continuous function $\varphi:[0, \infty) \rightarrow[0, \infty)$, with $\varphi(0)=0$, such that for each $x, y \in E$,

$$
\|T x-T y\| \leq \delta\|x-y\|+\varphi(\|x-T x\|) .
$$

A map satisfying (1.4) need not have a fixed point, even if $E$ is complete. For example, let $E=[0, \infty)$ and define $T$ by

$$
T x= \begin{cases}1.0, & 0 \leq x \leq 0.8 \\ 0.6, & 0.8<x\end{cases}
$$

WLOG, assume that $x<y$. Then, for $0 \leq x<y \leq 0.8$ or $0.8<x<y,\|T x-T y\|=0$, and (1.4) is automatically satisfied. 
If $0 \leq x \leq 0.8<y$, then $\|T x-T y\|=0.4$.

Define $\varphi$ by $\varphi(t)=L t$ for any $L \geq 2$. Then $\varphi$ is increasing, continuous, and $\varphi(0)=0$. Also, $\|x-T x\|=1-x$ so that $\varphi(\|x-T x\|)=L(1-x) \geq 0.2 L \geq 0.4$.

Therefore

$$
0.4=\|T x-T y\| \leq L\|x-T x\| \leq \delta\|x-y\|+L\|x-T x\|
$$

for any $0 \leq \delta<1$, and (1.4) is satisfied for $0 \leq x \leq 0.8<y$. But $T$ has no fixed point.

However, using (1.4) it is obvious that if $T$ has a fixed point, then it is unique.

From now on, we demand that $\mathbb{N}$ denotes the set of all nonnegative integers. Let $X$ be a Banach space, let $E \subset X$ be a nonempty closed, convex subset of $X$, and let $T$ be a selfmap on $E$. Define $F_{T}:=\{p \in X: p=T p\}$ to be the set of fixed points of $T$. Let $\left\{\alpha_{n}\right\}_{n=0}^{\infty}$, $\left\{\beta_{n}\right\}_{n=0}^{\infty},\left\{\gamma_{n}\right\}_{n=0}^{\infty}$ and $\left\{\beta_{n}^{i}\right\}_{n=0}^{\infty}, i=\overline{1, k-2}, k \geq 2$ be real sequences in $[0,1)$ satisfying certain conditions.

In [5] Rhoades and Şoltuz introduced a multi-step iterative procedure given by

$$
\left\{\begin{array}{l}
x_{0} \in E \\
y_{n}^{k-1}=\left(1-\beta_{n}^{k-1}\right) x_{n}+\beta_{n}^{k-1} T x_{n}, \quad k \geq 2, \\
y_{n}^{i}=\left(1-\beta_{n}^{i}\right) x_{n}+\beta_{n}^{i} T y_{n}^{i+1}, \quad i=\overline{1, k-2}, \\
x_{n+1}=\left(1-\alpha_{n}\right) x_{n}+\alpha_{n} T y_{n}^{1}, \quad n \in \mathbb{N} .
\end{array}\right.
$$

The sequence $\left\{x_{n}\right\}_{n=0}^{\infty}$ defined by

$$
\left\{\begin{array}{l}
x_{0} \in E, \\
x_{n+1}=\left(1-\alpha_{n}\right) T x_{n}+\alpha_{n} T y_{n}, \\
y_{n}=\left(1-\beta_{n}\right) x_{n}+\beta_{n} T x_{n}, \quad n \in \mathbb{N},
\end{array}\right.
$$

is known as the S-iteration process (see $[12,17,39])$.

Thianwan [6] defined a two-step iteration $\left\{x_{n}\right\}_{n=0}^{\infty}$ by

$$
\left\{\begin{array}{l}
x_{0} \in E, \\
x_{n+1}=\left(1-\alpha_{n}\right) y_{n}+\alpha_{n} T y_{n}, \\
y_{n}=\left(1-\beta_{n}\right) x_{n}+\beta_{n} T x_{n}, \quad n \in \mathbb{N} .
\end{array}\right.
$$

Recently Phuengrattana and Suantai [7] introduced an SP iteration method defined by

$$
\left\{\begin{array}{l}
x_{0} \in E, \\
x_{n+1}=\left(1-\alpha_{n}\right) y_{n}+\alpha_{n} T y_{n}, \\
y_{n}=\left(1-\beta_{n}\right) z_{n}+\beta_{n} T z_{n}, \\
z_{n}=\left(1-\gamma_{n}\right) x_{n}+\gamma_{n} T x_{n}, \quad n \in \mathbb{N} .
\end{array}\right.
$$


We shall employ the following iterative process. For an arbitrary fixed order $k \geq 2$,

$$
\left\{\begin{array}{l}
x_{0} \in E, \\
x_{n+1}=\left(1-\alpha_{n}\right) y_{n}^{1}+\alpha_{n} T y_{n}^{1}, \\
y_{n}^{1}=\left(1-\beta_{n}^{1}\right) y_{n}^{2}+\beta_{n}^{1} T y_{n}^{2}, \\
y_{n}^{2}=\left(1-\beta_{n}^{2}\right) y_{n}^{3}+\beta_{n}^{2} T y_{n}^{3}, \\
\cdots, \\
y_{n}^{k-2}=\left(1-\beta_{n}^{k-2}\right) y_{n}^{k-1}+\beta_{n}^{k-2} T y_{n}^{k-1}, \\
y_{n}^{k-1}=\left(1-\beta_{n}^{k-1}\right) x_{n}+\beta_{n}^{k-1} T x_{n}, \quad n \in \mathbb{N},
\end{array}\right.
$$

or, in short,

$$
\left\{\begin{array}{l}
x_{0} \in E \\
x_{n+1}=\left(1-\alpha_{n}\right) y_{n}^{1}+\alpha_{n} T y_{n}^{1}, \\
y_{n}^{i}=\left(1-\beta_{n}^{i}\right) y_{n}^{i+1}+\beta_{n}^{i} T y_{n}^{i+1}, \quad i=\overline{1, k-2} \\
y_{n}^{k-1}=\left(1-\beta_{n}^{k-1}\right) x_{n}+\beta_{n}^{k-1} T x_{n}, \quad n \in \mathbb{N}
\end{array}\right.
$$

where

$$
\left\{\alpha_{n}\right\}_{n=0}^{\infty} \subset[0,1), \quad \sum_{n=0}^{\infty} \alpha_{n}=\infty,
$$

and

$$
\left\{\beta_{n}^{i}\right\}_{n=0}^{\infty} \subset[0,1), \quad i=\overline{1, k-1} .
$$

Remark 1 If each $\gamma_{n}=0$, then SP iteration (1.8) reduces to two-step iteration (1.7). By taking $k=3$ and $k=2$ in (1.10), we obtain iterations (1.8) and (1.7), respectively.

We shall need the following definition and lemma in the sequel.

Definition 1 [40] Let $T, \widetilde{T}: X \rightarrow X$ be two operators. We say that $\widetilde{T}$ is an approximate operator for $T$ if, for some $\varepsilon>0$, we have

$$
\|T x-\widetilde{T} x\| \leq \varepsilon
$$

for all $x \in X$.

Lemma 1 [34] Let $\left\{a_{n}\right\}_{n=0}^{\infty}$ be a nonnegative sequence for which one assumes that there exists an $n_{0} \in \mathbb{N}$ such that for all $n \geq n_{0}$,

$$
a_{n+1} \leq\left(1-\mu_{n}\right) a_{n}+\mu_{n} \eta_{n}
$$

is satisfied, where $\mu_{n} \in(0,1)$ for all $n \in \mathbb{N}, \sum_{n=0}^{\infty} \mu_{n}=\infty$ and $\eta_{n} \geq 0, \forall n \in \mathbb{N}$. Then the following holds:

$$
0 \leq \lim _{n \rightarrow \infty} \sup a_{n} \leq \lim _{n \rightarrow \infty} \sup \eta_{n} .
$$




\section{Main results}

For simplicity we use the following notation throughout this section.

For any iterative process, $\left\{x_{n}\right\}_{n=0}^{\infty}$ and $\left\{u_{n}\right\}_{n=0}^{\infty}$ denote iterative sequences associated to $T$ and $\widetilde{T}$, respectively.

Theorem 1 Let $T: E \rightarrow E$ be a map satisfying (1.4) with $F_{T} \neq \emptyset$, and let $\left\{x_{n}\right\}_{n=0}^{\infty}$ be a sequence defined by (1.10), then the sequence $\left\{x_{n}\right\}_{n=0}^{\infty}$ converges to the unique fixed point of $T$.

Proof The proof can be easily obtained by using the argument in the proof of ([36], Theorem 3.1).

This result allows us to give the next theorem.

Theorem 2 Let $T: E \rightarrow E$ be a map satisfying (1.4) with $F_{T} \neq \emptyset$, and let $\widetilde{T}$ be an approximate operator of $T$ as in Definition 1 . Let $\left\{x_{n}\right\}_{n=0}^{\infty},\left\{u_{n}\right\}_{n=0}^{\infty}$ be two iterative sequences defined by (1.10) with real sequences $\left\{\alpha_{n}\right\}_{n=0}^{\infty},\left\{\beta_{n}^{i}\right\}_{n=0}^{\infty} \subset[0,1)$ satisfying (i) $0 \leq \beta_{n}^{i}<\alpha_{n} \leq 1$, $i=\overline{1, k-1}$, (ii) $\sum \alpha_{n}=\infty$. If $p=$ Tp and $q=\widetilde{T} q$, then we have

$$
\|p-q\| \leq \frac{k \varepsilon}{1-\delta}
$$

Proof For given $x_{0} \in E$ and $u_{0} \in E$, we consider the following multi-step iteration for $T$ and $\widetilde{T}$ :

$$
\left\{\begin{array}{l}
x_{0} \in E \\
x_{n+1}=\left(1-\alpha_{n}\right) y_{n}^{1}+\alpha_{n} T y_{n}^{1}, \\
y_{n}^{i}=\left(1-\beta_{n}^{i}\right) y_{n}^{i+1}+\beta_{n}^{i} T y_{n}^{i+1}, \quad i=\overline{1, k-2}, \\
y_{n}^{k-1}=\left(1-\beta_{n}^{k-1}\right) x_{n}+\beta_{n}^{k-1} T x_{n}, \quad k \geq 2, n \in \mathbb{N},
\end{array}\right.
$$

and

$$
\left\{\begin{array}{l}
u_{0} \in E, \\
u_{n+1}=\left(1-\alpha_{n}\right) v_{n}^{1}+\alpha_{n} \widetilde{T} v_{n}^{1}, \\
v_{n}^{i}=\left(1-\beta_{n}^{i}\right) v_{n}^{i+1}+\beta_{n}^{i} \widetilde{T} v_{n}^{i+1}, \quad i=\overline{1, k-2}, \\
v_{n}^{k-1}=\left(1-\beta_{n}^{k-1}\right) u_{n}+\beta_{n}^{k-1} \widetilde{T} u_{n}, \quad k \geq 2, n \in \mathbb{N} .
\end{array}\right.
$$

Thus, from (1.4), (2.1) and (2.2), we have the following inequalities.

$$
\begin{aligned}
\left\|x_{n+1}-u_{n+1}\right\| & =\left\|\left(1-\alpha_{n}\right)\left(y_{n}^{1}-v_{n}^{1}\right)+\alpha_{n}\left(T y_{n}^{1}-\widetilde{T} v_{n}^{1}\right)\right\| \\
& \leq\left(1-\alpha_{n}\right)\left\|y_{n}^{1}-v_{n}^{1}\right\|+\alpha_{n}\left\|T y_{n}^{1}-\widetilde{T} v_{n}^{1}\right\| \\
& =\left(1-\alpha_{n}\right)\left\|y_{n}^{1}-v_{n}^{1}\right\|+\alpha_{n}\left\|T y_{n}^{1}-T v_{n}^{1}+T v_{n}^{1}-\widetilde{T} v_{n}^{1}\right\| \\
& \leq\left(1-\alpha_{n}\right)\left\|y_{n}^{1}-v_{n}^{1}\right\|+\alpha_{n}\left\|T y_{n}^{1}-T v_{n}^{1}\right\|+\alpha_{n}\left\|T v_{n}^{1}-\widetilde{T} v_{n}^{1}\right\| \\
& \leq\left(1-\alpha_{n}\right)\left\|y_{n}^{1}-v_{n}^{1}\right\|+\alpha_{n} \delta\left\|y_{n}^{1}-v_{n}^{1}\right\|+\alpha_{n} \varphi\left(\left\|y_{n}^{1}-T y_{n}^{1}\right\|\right)+\alpha_{n} \varepsilon \\
& =\left[1-\alpha_{n}(1-\delta)\right]\left\|y_{n}^{1}-v_{n}^{1}\right\|+\alpha_{n} \varphi\left(\left\|y_{n}^{1}-T y_{n}^{1}\right\|\right)+\alpha_{n} \varepsilon,
\end{aligned}
$$




$$
\begin{aligned}
\left\|y_{n}^{1}-v_{n}^{1}\right\| & =\left\|\left(1-\beta_{n}^{1}\right)\left(y_{n}^{2}-v_{n}^{2}\right)+\beta_{n}^{1}\left(T y_{n}^{2}-\widetilde{T} v_{n}^{2}\right)\right\| \\
& \leq\left(1-\beta_{n}^{1}\right)\left\|y_{n}^{2}-v_{n}^{2}\right\|+\beta_{n}^{1}\left\|T y_{n}^{2}-\widetilde{T} v_{n}^{2}\right\| \\
& \leq\left(1-\beta_{n}^{1}\right)\left\|y_{n}^{2}-v_{n}^{2}\right\|+\beta_{n}^{1}\left\|T y_{n}^{2}-T v_{n}^{2}\right\|+\beta_{n}^{1}\left\|T v_{n}^{2}-\widetilde{T} v_{n}^{2}\right\| \\
& \leq\left(1-\beta_{n}^{1}\right)\left\|y_{n}^{2}-v_{n}^{2}\right\|+\beta_{n}^{1} \delta\left\|y_{n}^{2}-v_{n}^{2}\right\|+\beta_{n}^{1} \varphi\left(\left\|y_{n}^{2}-T y_{n}^{2}\right\|\right)+\beta_{n}^{1} \varepsilon \\
& =\left[1-\beta_{n}^{1}(1-\delta)\right]\left\|y_{n}^{2}-v_{n}^{2}\right\|+\beta_{n}^{1} \varphi\left(\left\|y_{n}^{2}-T y_{n}^{2}\right\|\right)+\beta_{n}^{1} \varepsilon, \\
\left\|y_{n}^{2}-v_{n}^{2}\right\| & =\left\|\left(1-\beta_{n}^{2}\right)\left(y_{n}^{3}-v_{n}^{3}\right)+\beta_{n}^{2}\left(T y_{n}^{3}-\widetilde{T} v_{n}^{3}\right)\right\| \\
& \leq\left(1-\beta_{n}^{2}\right)\left\|y_{n}^{3}-v_{n}^{3}\right\|+\beta_{n}^{2}\left\|T y_{n}^{3}-\widetilde{T} v_{n}^{3}\right\| \\
& \leq\left(1-\beta_{n}^{2}\right)\left\|y_{n}^{3}-v_{n}^{3}\right\|+\beta_{n}^{2}\left\|T y_{n}^{3}-T v_{n}^{3}\right\|+\beta_{n}^{2}\left\|T v_{n}^{3}-\widetilde{T} v_{n}^{3}\right\| \\
& \leq\left(1-\beta_{n}^{2}\right)\left\|y_{n}^{3}-v_{n}^{3}\right\|+\beta_{n}^{2} \delta\left\|y_{n}^{3}-v_{n}^{3}\right\|+\beta_{n}^{2} \varphi\left(\left\|y_{n}^{3}-T y_{n}^{3}\right\|\right)+\beta_{n}^{2} \varepsilon \\
& =\left[1-\beta_{n}^{2}(1-\delta)\right]\left\|y_{n}^{3}-v_{n}^{3}\right\|+\beta_{n}^{2} \varphi\left(\left\|y_{n}^{3}-T y_{n}^{3}\right\|\right)+\beta_{n}^{2} \varepsilon .
\end{aligned}
$$

Combining (2.3), (2.4) and (2.5), we obtain

$$
\begin{aligned}
\left\|x_{n+1}-u_{n+1}\right\| \leq & {\left[1-\alpha_{n}(1-\delta)\right]\left[1-\beta_{n}^{1}(1-\delta)\right]\left[1-\beta_{n}^{2}(1-\delta)\right]\left\|y_{n}^{3}-v_{n}^{3}\right\| } \\
& +\left[1-\alpha_{n}(1-\delta)\right]\left[1-\beta_{n}^{1}(1-\delta)\right] \beta_{n}^{2} \varphi\left(\left\|y_{n}^{3}-T y_{n}^{3}\right\|\right) \\
& +\left[1-\alpha_{n}(1-\delta)\right]\left[1-\beta_{n}^{1}(1-\delta)\right] \beta_{n}^{2} \varepsilon \\
& +\left[1-\alpha_{n}(1-\delta)\right] \beta_{n}^{1} \varphi\left(\left\|y_{n}^{2}-T y_{n}^{2}\right\|\right) \\
& +\left[1-\alpha_{n}(1-\delta)\right] \beta_{n}^{1} \varepsilon+\alpha_{n} \varphi\left(\left\|y_{n}^{1}-T y_{n}^{1}\right\|\right)+\alpha_{n} \varepsilon .
\end{aligned}
$$

Thus, by induction, we get

$$
\begin{aligned}
\left\|x_{n+1}-u_{n+1}\right\| \leq & {\left[1-\alpha_{n}(1-\delta)\right] } \\
& {\left[1-\beta_{n}^{1}(1-\delta)\right] \cdots\left[1-\beta_{n}^{k-2}(1-\delta)\right]\left\|y_{n}^{k-1}-v_{n}^{k-1}\right\| } \\
& +\left[1-\alpha_{n}(1-\delta)\right] \\
& {\left[1-\beta_{n}^{1}(1-\delta)\right] \cdots\left[1-\beta_{n}^{k-3}(1-\delta)\right] \beta_{n}^{k-2} \varphi\left(\left\|y_{n}^{k-1}-T y_{n}^{k-1}\right\|\right) } \\
& +\cdots+\left[1-\alpha_{n}(1-\delta)\right] \beta_{n}^{1} \varphi\left(\left\|y_{n}^{2}-T y_{n}^{2}\right\|\right)+\alpha_{n} \varphi\left(\left\|y_{n}^{1}-T y_{n}^{1}\right\|\right) \\
& +\left[1-\alpha_{n}(1-\delta)\right]\left[1-\beta_{n}^{1}(1-\delta)\right] \cdots\left[1-\beta_{n}^{k-3}(1-\delta)\right] \beta_{n}^{k-2} \varepsilon \\
& +\cdots+\left[1-\alpha_{n}(1-\delta)\right] \beta_{n}^{1} \varepsilon+\alpha_{n} \varepsilon .
\end{aligned}
$$

Again, using (1.4), (2.1) and (2.2), we get

$$
\begin{aligned}
\left\|y_{n}^{k-1}-v_{n}^{k-1}\right\| & =\left\|\left(1-\beta_{n}^{k-1}\right)\left(x_{n}-u_{n}\right)+\beta_{n}^{k-1}\left(T x_{n}-\widetilde{T} u_{n}\right)\right\| \\
& \leq\left(1-\beta_{n}^{k-1}\right)\left\|x_{n}-u_{n}\right\|+\beta_{n}^{k-1}\left\|T x_{n}-\widetilde{T} u_{n}\right\| \\
& \leq\left(1-\beta_{n}^{k-1}\right)\left\|x_{n}-u_{n}\right\|+\beta_{n}^{k-1}\left\|T x_{n}-T u_{n}\right\|+\beta_{n}^{k-1}\left\|T u_{n}-\widetilde{T} u_{n}\right\| \\
& \leq\left[1-\beta_{n}^{k-1}(1-\delta)\right]\left\|x_{n}-u_{n}\right\|+\beta_{n}^{k-1} \varphi\left(\left\|x_{n}-T x_{n}\right\|\right)+\beta_{n}^{k-1} \varepsilon .
\end{aligned}
$$


Substituting (2.8) in (2.7), we have

$$
\begin{aligned}
\left\|x_{n+1}-u_{n+1}\right\| \leq & {\left[1-\alpha_{n}(1-\delta)\right] } \\
& {\left[1-\beta_{n}^{1}(1-\delta)\right] \cdots\left[1-\beta_{n}^{k-1}(1-\delta)\right]\left\|x_{n}-u_{n}\right\| } \\
& +\left[1-\alpha_{n}(1-\delta)\right] \\
& {\left[1-\beta_{n}^{1}(1-\delta)\right] \cdots\left[1-\beta_{n}^{k-2}(1-\delta)\right] \beta_{n}^{k-1} \varphi\left(\left\|x_{n}-T x_{n}\right\|\right) } \\
& +\left[1-\alpha_{n}(1-\delta)\right] \\
& {\left[1-\beta_{n}^{1}(1-\delta)\right] \cdots\left[1-\beta_{n}^{k-3}(1-\delta)\right] \beta_{n}^{k-2} \varphi\left(\left\|y_{n}^{k-1}-T y_{n}^{k-1}\right\|\right) } \\
& +\cdots+\left[1-\alpha_{n}(1-\delta)\right] \beta_{n}^{1} \varphi\left(\left\|y_{n}^{2}-T y_{n}^{2}\right\|\right)+\alpha_{n} \varphi\left(\left\|y_{n}^{1}-T y_{n}^{1}\right\|\right) \\
& +\left[1-\alpha_{n}(1-\delta)\right]\left[1-\beta_{n}^{1}(1-\delta)\right] \cdots\left[1-\beta_{n}^{k-2}(1-\delta)\right] \beta_{n}^{k-1} \varepsilon \\
& +\cdots+\left[1-\alpha_{n}(1-\delta)\right] \beta_{n}^{1} \varepsilon+\alpha_{n} \varepsilon .
\end{aligned}
$$

Since $\delta \in[0,1)$ and $\left\{\alpha_{n}\right\}_{n=0}^{\infty},\left\{\beta_{n}^{i}\right\}_{n=0}^{\infty} \subset[0,1)$, for $i=\overline{1, k-1}$, we have

$$
\left[1-\alpha_{n}(1-\delta)\right]\left[1-\beta_{n}^{1}(1-\delta)\right] \cdots\left[1-\beta_{n}^{i}(1-\delta)\right] \leq\left[1-\alpha_{n}(1-\delta)\right] .
$$

From inequality (2.10) and assumption (i) in (2.9), it follows

$$
\begin{aligned}
\left\|x_{n+1}-u_{n+1}\right\| \leq & {\left[1-\alpha_{n}(1-\delta)\right]\left\|x_{n}-u_{n}\right\| } \\
& +\alpha_{n} \varphi\left(\left\|x_{n}-T x_{n}\right\|\right)+\alpha_{n} \varphi\left(\left\|y_{n}^{k-1}-T y_{n}^{k-1}\right\|\right) \\
& +\cdots+\alpha_{n} \varphi\left(\left\|y_{n}^{2}-T y_{n}^{2}\right\|\right)+\alpha_{n} \varphi\left(\left\|y_{n}^{1}-T y_{n}^{1}\right\|\right) \\
& +\alpha_{n} \varepsilon+\alpha_{n} \varepsilon+\cdots+\alpha_{n} \varepsilon+\alpha_{n} \varepsilon \\
= & {\left[1-\alpha_{n}(1-\delta)\right]\left\|x_{n}-u_{n}\right\| } \\
& +\alpha_{n}(1-\delta)\left\{\frac{\varphi\left(\left\|x_{n}-T x_{n}\right\|\right)+\varphi\left(\left\|y_{n}^{k-1}-T y_{n}^{k-1}\right\|\right)}{1-\delta}\right. \\
& \left.+\cdots+\frac{\varphi\left(\left\|y_{n}^{1}-T y_{n}^{1}\right\|\right)+k \varepsilon}{1-\delta}\right\} .
\end{aligned}
$$

Define

$$
\begin{aligned}
& a_{n}:=\left\|x_{n}-u_{n}\right\|, \\
& \mu_{n}:=\alpha_{n}(1-\delta) \in(0.1), \\
& \eta_{n}:=\left\{\frac{\varphi\left(\left\|x_{n}-T x_{n}\right\|\right)+\varphi\left(\left\|y_{n}^{k-1}-T y_{n}^{k-1}\right\|\right)}{1-\delta}+\cdots+\frac{\varphi\left(\left\|y_{n}^{1}-T y_{n}^{1}\right\|\right)+k \varepsilon}{1-\delta}\right\} .
\end{aligned}
$$

From Theorem 1 it follows that $\lim _{n \rightarrow \infty}\left\|x_{n}-p\right\|=0$. Since $T$ satisfies condition (1.4) and $T p=p \in F_{T}$,

$$
\begin{aligned}
0 & \leq\left\|x_{n}-T x_{n}\right\| \\
& \leq\left\|x_{n}-p\right\|+\left\|T p-T x_{n}\right\|
\end{aligned}
$$




$$
\begin{aligned}
& \leq\left\|x_{n}-p\right\|+\delta\left\|p-x_{n}\right\|+\varphi(\|p-T p\|) \\
& =(1+\delta)\left\|x_{n}-p\right\| \rightarrow 0 \quad \text { as } n \rightarrow \infty
\end{aligned}
$$

Since $\beta_{n}^{i} \in[0,1), \forall n \in \mathbb{N}, i=\overline{1, k-1}$, using (1.4) and (1.10), we have

$$
\begin{aligned}
0 & \leq\left\|y_{n}^{1}-T y_{n}^{1}\right\|=\left\|y_{n}^{1}-p+p-T y_{n}^{1}\right\| \\
& \leq\left\|y_{n}^{1}-p\right\|+\left\|T p-T y_{n}^{1}\right\| \\
& \leq\left\|y_{n}^{1}-p\right\|+\delta\left\|p-y_{n}^{1}\right\|+\varphi(\|p-T p\|) \\
& =(1+\delta)\left\|y_{n}^{1}-p\right\| \\
& =(1+\delta)\left\|\left(1-\beta_{n}^{1}\right) y_{n}^{2}+\beta_{n}^{1} T y_{n}^{2}-p\left(1-\beta_{n}^{1}+\beta_{n}^{1}\right)\right\| \\
& \leq(1+\delta)\left\{\left(1-\beta_{n}^{1}\right)\left\|y_{n}^{2}-p\right\|+\beta_{n}^{1}\left\|T y_{n}^{2}-T p\right\|\right\} \\
& \leq(1+\delta)\left\{\left(1-\beta_{n}^{1}\right)\left\|y_{n}^{2}-p\right\|+\beta_{n}^{1} \delta\left\|y_{n}^{2}-p\right\|\right\} \\
& =(1+\delta)\left[1-\beta_{n}^{1}(1-\delta)\right]\left\|y_{n}^{2}-p\right\| \\
& \leq \cdots \\
& \leq(1+\delta)\left[1-\beta_{n}^{1}(1-\delta)\right] \cdots\left[1-\beta_{n}^{k-2}(1-\delta)\right]\left\|y_{n}^{k-1}-p\right\| \\
& \leq(1+\delta)\left[1-\beta_{n}^{1}(1-\delta)\right] \cdots\left[1-\beta_{n}^{k-1}(1-\delta)\right]\left\|x_{n}-p\right\| \\
& \leq(1+\delta)\left\|x_{n}-p\right\| \rightarrow 0 \quad \text { as } n \rightarrow \infty .
\end{aligned}
$$

It is easy to see from (2.13) that this result is also valid for $\left\|T y_{n}^{2}-y_{n}^{2}\right\|, \ldots,\left\|T y_{n}^{k-1}-y_{n}^{k-1}\right\|$.

Since $\varphi$ is continuous, we have

$$
\lim _{n \rightarrow \infty} \varphi\left(\left\|x_{n}-T x_{n}\right\|\right)=\lim _{n \rightarrow \infty} \varphi\left(\left\|y_{n}^{1}-T y_{n}^{1}\right\|\right)=\cdots=\lim _{n \rightarrow \infty} \varphi\left(\left\|y_{n}^{k-1}-T y_{n}^{k-1}\right\|\right)=0
$$

Hence an application of Lemma 1 to (2.11) leads to

$$
\|p-q\| \leq \frac{k \varepsilon}{1-\delta}
$$

As shown by Hussain et al. ([22], Theorem 8), in an arbitrary Banach space $X$, the Siteration $\left\{x_{n}\right\}_{n=0}^{\infty}$ given by (1.6) converges to the fixed point of $T$, where $T: E \rightarrow E$ is a mapping satisfying condition (1.3).

Theorem 3 Let $T: E \rightarrow E$ be a map satisfying (1.4) with $F_{T} \neq \emptyset$, and let $\left\{x_{n}\right\}_{n=0}^{\infty}$ be defined by (1.6) with real sequences $\left\{\beta_{n}\right\}_{n=0}^{\infty},\left\{\alpha_{n}\right\}_{n=0}^{\infty} \subset[0,1)$ satisfying $\sum_{n=0}^{\infty} \alpha_{n}=\infty$. Then the sequence $\left\{x_{n}\right\}_{n=0}^{\infty}$ converges to the unique fixed point of $T$.

Proof The argument is similar to the proof of Theorem 8 of [22], and is thus omitted.

We now prove the result on data dependence for the S-iterative procedure by utilizing Theorem 3. 
Theorem 4 Let $T, \widetilde{T}$ be two operators as in Theorem 2. Let $\left\{x_{n}\right\}_{n=0}^{\infty},\left\{u_{n}\right\}_{n=0}^{\infty}$ be S-iterations defined by (1.6) with real sequences $\left\{\beta_{n}\right\}_{n=0}^{\infty},\left\{\alpha_{n}\right\}_{n=0}^{\infty} \subset[0,1)$ satisfying (i) $\frac{1}{2} \leq \alpha_{n}, \forall n \in \mathbb{N}$, and (ii) $\sum_{n=0}^{\infty} \alpha_{n}=\infty$. If $p=$ Tp and $q=\widetilde{T} q$, then we have

$$
\|p-q\| \leq \frac{3 \varepsilon}{1-\delta}
$$

Proof For a given $x_{0} \in E$ and $u_{0} \in E$, we consider the following iteration for $T$ and $\widetilde{T}$ :

$$
\left\{\begin{array}{l}
x_{0} \in E, \\
x_{n+1}=\left(1-\alpha_{n}\right) T x_{n}+\alpha_{n} T y_{n}, \\
y_{n}=\left(1-\beta_{n}\right) x_{n}+\beta_{n} T x_{n}, \quad n \in \mathbb{N}
\end{array}\right.
$$

and

$$
\left\{\begin{array}{l}
u_{0} \in E, \\
u_{n+1}=\left(1-\alpha_{n}\right) \widetilde{T} u_{n}+\alpha_{n} \widetilde{T} v_{n}, \\
v_{n}=\left(1-\beta_{n}\right) u_{n}+\beta_{n} \widetilde{T} u_{n}, \quad n \in \mathbb{N} .
\end{array}\right.
$$

Using (1.4), (2.16) and (2.17), we obtain the following estimates:

$$
\begin{aligned}
\left\|x_{n+1}-u_{n+1}\right\|= & \left\|\left(1-\alpha_{n}\right)\left(T x_{n}-\widetilde{T} u_{n}\right)+\alpha_{n}\left(T y_{n}-\widetilde{T} v_{n}\right)\right\| \\
\leq & \left(1-\alpha_{n}\right)\left\|T x_{n}-\widetilde{T} u_{n}\right\|+\alpha_{n}\left\|T y_{n}-\widetilde{T} v_{n}\right\| \\
= & \left(1-\alpha_{n}\right)\left\|T x_{n}-T u_{n}+T u_{n}-\widetilde{T} u_{n}\right\|+\alpha_{n}\left\|T y_{n}-T v_{n}+T v_{n}-\widetilde{T} v_{n}\right\| \\
\leq & \left(1-\alpha_{n}\right)\left\{\left\|T x_{n}-T u_{n}\right\|+\left\|T u_{n}-\widetilde{T} u_{n}\right\|\right\} \\
& \quad+\alpha_{n}\left\{\left\|T y_{n}-T v_{n}\right\|+\left\|T v_{n}-\widetilde{T} v_{n}\right\|\right\} \\
\leq & \left(1-\alpha_{n}\right)\left\{\delta\left\|x_{n}-u_{n}\right\|+\varphi\left(\left\|x_{n}-T x_{n}\right\|\right)+\varepsilon\right\} \\
& \quad+\alpha_{n}\left\{\delta\left\|y_{n}-v_{n}\right\|+\varphi\left(\left\|y_{n}-T y_{n}\right\|\right)+\varepsilon\right\}, \\
\left\|y_{n}-v_{n}\right\|= & \left\|\left(1-\beta_{n}\right)\left(x_{n}-u_{n}\right)+\beta_{n}\left(T x_{n}-\widetilde{T} u_{n}\right)\right\| \\
\leq & \left(1-\beta_{n}\right)\left\|x_{n}-u_{n}\right\|+\beta_{n}\left\|T x_{n}-\widetilde{T} u_{n}\right\| \\
= & \left(1-\beta_{n}\right)\left\|x_{n}-u_{n}\right\|+\beta_{n}\left\|T x_{n}-T u_{n}+T u_{n}-\widetilde{T} u_{n}\right\| \\
\leq & \left(1-\beta_{n}\right)\left\|x_{n}-u_{n}\right\|+\beta_{n}\left\{\left\|T x_{n}-T u_{n}\right\|+\left\|T u_{n}-\widetilde{T} u_{n}\right\|\right\} \\
\leq & \left(1-\beta_{n}\right)\left\|x_{n}-u_{n}\right\|+\beta_{n}\left\{\delta\left\|x_{n}-u_{n}\right\|+\varphi\left(\left\|x_{n}-T x_{n}\right\|\right)+\varepsilon\right\} \\
= & {\left[1-\beta_{n}(1-\delta)\right]\left\|x_{n}-u_{n}\right\|+\beta_{n} \varphi\left(\left\|x_{n}-T x_{n}\right\|\right)+\beta_{n} \varepsilon . }
\end{aligned}
$$

Combining (2.18) and (2.19), we get

$$
\begin{aligned}
\left\|x_{n+1}-u_{n+1}\right\| \leq & \left\{\left(1-\alpha_{n}\right) \delta+\alpha_{n} \delta\left[1-\beta_{n}(1-\delta)\right]\right\}\left\|x_{n}-u_{n}\right\| \\
& +\left\{1-\alpha_{n}+\alpha_{n} \delta \beta_{n}\right\} \varphi\left(\left\|x_{n}-T x_{n}\right\|\right)+\alpha_{n} \varphi\left(\left\|y_{n}-T y_{n}\right\|\right) \\
& +\alpha_{n} \delta \beta_{n} \varepsilon+\left(1-\alpha_{n}\right) \varepsilon+\alpha_{n} \varepsilon
\end{aligned}
$$


For $\left\{\alpha_{n}\right\}_{n=0}^{\infty},\left\{\beta_{n}\right\}_{n=0}^{\infty} \subset[0,1)$ and $\delta \in[0,1)$,

$$
\left(1-\alpha_{n}\right) \delta<1-\alpha_{n}, \quad 1-\beta_{n}(1-\delta)<1, \quad \alpha_{n} \delta \beta_{n}<\alpha_{n}
$$

It follows from assumption (i) that

$$
1-\alpha_{n}<\alpha_{n}, \quad \forall n \in \mathbb{N} \text {. }
$$

Therefore, combining (2.22) and (2.21) to (2.20) gives

$$
\begin{aligned}
\left\|x_{n+1}-u_{n+1}\right\| \leq & {\left[1-\alpha_{n}(1-\delta)\right]\left\|x_{n}-u_{n}\right\| } \\
& +2 \alpha_{n} \varphi\left(\left\|x_{n}-T x_{n}\right\|\right)+\alpha_{n} \varphi\left(\left\|y_{n}-T y_{n}\right\|\right) \\
& +\alpha_{n} \varepsilon+\alpha_{n} \varepsilon+\alpha_{n} \varepsilon
\end{aligned}
$$

or, equivalently,

$$
\begin{aligned}
&\left\|x_{n+1}-u_{n+1}\right\| \leq\left[1-\alpha_{n}(1-\delta)\right]\left\|x_{n}-u_{n}\right\| \\
&+\alpha_{n}(1-\delta) \frac{\left\{2 \varphi\left(\left\|x_{n}-T x_{n}\right\|\right)+\varphi\left(\left\|y_{n}-T y_{n}\right\|\right)+3 \varepsilon\right\}}{1-\delta} .
\end{aligned}
$$

Now define

$$
\begin{aligned}
& a_{n}:=\left\|x_{n}-u_{n}\right\|, \\
& \mu_{n}:=\alpha_{n}(1-\delta) \in(0,1) \\
& \eta_{n}:=\frac{2 \varphi\left(\left\|x_{n}-T x_{n}\right\|\right)+\varphi\left(\left\|y_{n}-T y_{n}\right\|\right)+3 \varepsilon}{1-\delta} .
\end{aligned}
$$

From Theorem 3, we have $\lim _{n \rightarrow \infty}\left\|x_{n}-p\right\|=0$. Since $T$ satisfies condition (1.4), and $T p=$ $p \in F_{T}$, using an argument similar to that in the proof of Theorem 2 ,

$$
\lim _{n \rightarrow \infty}\left\|x_{n}-T x_{n}\right\|=\lim _{n \rightarrow \infty}\left\|y_{n}-T y_{n}\right\|=0
$$

Using the fact that $\varphi$ is continuous, we have

$$
\lim _{n \rightarrow \infty} \varphi\left(\left\|x_{n}-T x_{n}\right\|\right)=\lim _{n \rightarrow \infty} \varphi\left(\left\|y_{n}-T y_{n}\right\|\right)=0
$$

An application of Lemma 1 to (2.24) leads to

$$
\|p-q\| \leq \frac{3 \varepsilon}{1-\delta}
$$

\section{Conclusion}

Since the iterative schemes (1.7) and (1.8) are special cases of the iterative process (1.10), Theorem 1 generalizes Theorem 2.1 of [19] and Theorem 2.1 of [18]. By taking $k=3$ and $k=2$ in Theorem 2, data dependence results for the iterative schemes (1.8) and (1.7) can be easily obtained. For $k=3$, Theorem 2 reduces to Theorem 3.2 of [35]. Since condition (1.4) is more general than condition (1.3), Theorem 3 generalizes Theorem 8 of [22]. 


\section{Competing interests}

The authors declare that they have no competing interests.

\section{Authors' contributions}

All authors contributed equally and significantly in writing this article. All authors read and approved the final manuscript.

\section{Author details}

'Department of Mathematics, Faculty of Science and Letters, Yildiz Technical University, Davutpasa Campus, Esenler, Istanbul, 34220, Turkey. ${ }^{2}$ Department of Mathematical Engineering, Faculty of Chemical and Metallurgical Engineering, Yildiz Technical University, Davutpasa Campus, Esenler, Istanbul, 34210, Turkey. ${ }^{3}$ Department of Mathematics, Indiana University, Bloomington, IN 47405-7106, USA.

\section{Acknowledgements}

The first two authors would like to thank Yıldız Technical University Scientific Research Projects Coordination Unit under project number BAPK 2012-07-03-DOP02 for financial support during the preparation of this manuscript.

\section{Received: 5 December 2012 Accepted: 10 March 2013 Published: 28 March 2013}

\section{References}

1. Rhoades, BE: A comparison of various definitions of contractive mappings. Trans. Am. Math. Soc. 226, 257-290 (1977)

2. Mann, WR: Mean value methods in iterations. Proc. Am. Math. Soc. 4, 506-510 (1953)

3. Ishikawa, S: Fixed points by a new iteration method. Proc. Am. Math. Soc. 44, 147-150 (1974)

4. Noor, MA: New approximation schemes for general variational inequalities. J. Math. Anal. Appl. 251, 217-229 (2000)

5. Rhoades, BE, Şoltuz, SM: The equivalence between Mann-Ishikawa iterations and multistep iteration. Nonlinear Anal. $58,219-228(2004)$

6. Thianwan, $\mathrm{S}$ : Common fixed points of new iterations for two asymptotically nonexpansive nonself mappings in a Banach space. J. Comput. Appl. Math. (2008). doi:10.1016/j.cam.2008.05.051

7. Phuengrattana, W, Suantai, S: On the rate of convergence of Mann, Ishikawa, Noor and SP iterations for continuous functions on an arbitrary interval. J. Comput. Appl. Math. 235, 3006-3014 (2011)

8. Glowinski, R, Le Tallec, P: Augmented Langrangian and Operator Splitting Methods in Nonlinear Mechanics. SIAM, Philadelphia (1989)

9. $\mathrm{Xu}, \mathrm{B}, \mathrm{Noor}, \mathrm{MA}$ : Ishikawa and Mann iteration process with errors for nonlinear strongly accretive operator equations. J. Math. Anal. Appl. 224, 91-101 (1998)

10. Takahashi, W: Iterative methods for approximation of fixed points and their applications. J. Oper. Res. Soc. Jpn. 43, 87-108 (2000)

11. Das, G, Debata, JP: Fixed points of quasi-nonexpansive mappings. Indian J. Pure Appl. Math. 17, 1263-1269 (1986)

12. Agarwal, RP, O'Regan, D, Sahu, DR: Iterative construction of fixed points of nearly asymptotically nonexpansive mappings. J. Nonlinear Convex Anal. 8, 61-79 (2007)

13. Berinde, $\mathrm{V}$ : On the convergence of the Ishikawa iteration in the class of quasi contractive operators. Acta Math. Univ. Comen. 73, 119-126 (2004)

14. Chidume, CE, Chidume, CO: Convergence theorem for fixed points of uniformly continuous generalized phihemicontractive mappings. J. Math. Anal. Appl. 303, 545-554 (2005)

15. Chidume, CE, Chidume, CO: Iterative approximation of fixed points of nonexpansive mappings. J. Math. Anal. Appl. 318, 288-295 (2006)

16. Suantai, S: Weak and strong convergence criteria of Noor iterations for asymptotically nonexpansive mappings. J. Math. Anal. Appl. 311, 506-517 (2005)

17. Sahu, DR: Applications of the S-iteration process to constrained minimization problems and split feasibility problems. Fixed Point Theory Appl. 12, 187-204 (2011)

18. Yıldıım, I, Özdemir, M, KızItunç, H: On the convergence of a new two-step iteration in the class of quasi-contractive operators. Int. J. Math. Anal. 3, 1881-1892 (2009)

19. Chugh, R, Kumar, V: Strong convergence of SP iterative scheme for quasi-contractive operators in Banach spaces. Int. J. Comput. Appl. 31, 21-27 (2011)

20. Osilike, MO, Udomene, A: Short proofs of stability results for fixed point iteration procedures for a class of contractive-type mappings. Indian J. Pure Appl. Math. 30, 1229-1234 (1999)

21. Rafiq, A: On the convergence of the three step iteration process in the class of quasi-contractive operators. Acta Math. Acad. Paedagog. Nyházi. 22, 305-309 (2006)

22. Hussain, N, Rafiq, A, Damjanović, B, Lazović, R: On rate of convergence of various iterative schemes. Fixed Point Theory Appl. 2011, Article ID 45 (2011). doi:10.1186/1687-1812-2011-45

23. Rus, IA, Muresan, S: Data dependence of the fixed points set of weakly Picard operators. Stud. Univ. Babeş-Bolyai, Math. 43, 79-83 (1998)

24. Rus, IA, Petruşel, A, Sîntamarian, A: Data dependence of the fixed points set of multivalued weakly Picard operators. Stud. Univ. Babeș-Bolyai, Math. 46,111-121 (2001)

25. Rus, IA, Petruşel, A, Sîntamarian, A: Data dependence of the fixed point set of some multivalued weakly Picard operators. Nonlinear Anal., Theory Methods Appl. 52, 1947-1959 (2003)

26. Berinde, V: On the approximation of fixed points of weak contractive mappings. Carpath. J. Math. 19, 7-22 (2003)

27. Espínola, R, Petruşel, A: Existence and data dependence of fixed points for multivalued operators on gauge spaces. J. Math. Anal. Appl. 309, 420-432 (2005)

28. Markin, JT: Continuous dependence of fixed point sets. Proc. Am. Math. Soc. 38, 545-547 (1973)

29. Chifu, C, Petruşel, G: Existence and data dependence of fixed points and strict fixed points for contractive-type multivalued operators. Fixed Point Theory Appl. (2007). doi:10.1155/2007/34248

30. Olatinwo, MO: Some results on the continuous dependence of the fixed points in normed linear space. Fixed Point Theory Appl. 10, 151-157 (2009) 
31. Olatinwo, $\mathrm{MO}$ : On the continuous dependence of the fixed points for $(\varphi, \psi)$-contractive-type operators. Kragujev. J. Math. 34, 91-102 (2010)

32. Şoltuz, SM: Data dependence for Mann iteration. Octogon Math. Mag. 9, 825-828 (2001)

33. Soltuz, SM: Data dependence for Ishikawa iteration. Lect. Mat. 25, 149-155 (2004)

34. Şoltuz, SM, Grosan, T: Data dependence for Ishikawa iteration when dealing with contractive like operators. Fixed Point Theory Appl. 2008, Article ID 242916 (2008). doi:10.1155/2008/242916

35. Chugh, R, Kumar, V: Data dependence of Noor and SP iterative schemes when dealing with quasi-contractive operators. Int. J. Comput. Appl. 40, 41-46 (2011)

36. Akewe, $\mathrm{H}$ : Strong convergence and stability of Jungck-multistep-SP iteration for generalized contractive-like inequality operators. Adv. Nat. Sci. 5, 21-27 (2012)

37. Zamfirescu, T: Fix point theorems in metric spaces. Arch. Math. 23, 292-298 (1972)

38. Imoru, CO, Olantiwo, MO: On the stability of Picard and Mann iteration processes. Carpath. J. Math. 19, 155-160 (2003)

39. Agarwal, RP, O'Regan, D, Sahu, DR: Fixed Point Theory for Lipschitzian Type-Mappings with Applications. Springer, New York (2009)

40. Berinde, V: Iterative Approximation of Fixed Points. Springer, Berlin (2007)

doi:10.1186/1687-1812-2013-76

Cite this article as: Gürsoy et al.: Data dependence results of new multi-step and S-iterative schemes for contractive-like operators. Fixed Point Theory and Applications 2013 2013:76.

\section{Submit your manuscript to a SpringerOpen ${ }^{\circ}$ journal and benefit from:}

- Convenient online submission

Rigorous peer review

- Immediate publication on acceptance

- Open access: articles freely available online

- High visibility within the field

- Retaining the copyright to your article 